\title{
Microstructure and other short time-scale events in millisecond pulsars
}

\author{
V. A. Soglasnov \\ Astro Space Center of the Lebedev Physical Institute, \\ Profsoyuznaya 84/39, 117810 Moscow, Russia
}

\begin{abstract}
MSP have much more various short time-scale phenomena than "classical" microstructure in NP. Some of them have a long life-time and are observable not only in single pulses.
\end{abstract}

Microstructure, observed in some bright normal pulsars (NP), is a fine structure inside single pulses, with a very short time-scale $1 \div 1000 \mu s$, or 1 arcsec $\div 3$ arcmin in longitude. The latter corresponds to $1 \mu s \div 0.2 n s$ for millisecond pulsars (MSP). For this, and for many other reasons, microstructure observations of MSP are seemed rather difficult. However, recent progress in coherent dispersion removal technique makes it possible, so MSP were included, since the last year, into pulsar's microstructure researches, conducted in ASC, partially in collaboration with other observers in Italy, Canada and others.

Preliminary analysis of the obtained data has shown, that a short timescale phenomena are in millisecond pulsars much more various, then "classical" microstructure, observed in NP. They differ not only in a microstructure characteristic time, but also in other parameters, in particular, some of them have a long life-time and are observed as "micropulse flare" (MPF), when very short $(\sim 1 \mu s)$ flash of radio emission arises in the profile, flaming up to a very high brightness, then goes out. The event may last few minutes, without any drift or shift in longitude, following very precisely to the pulsar's period. Figure 1 demonstrates such flare, observed in the original MSP J1939+2134 at Meidicina (Sculachev et al, 1999).

Much more hard task is to investigate a "classical" short-lived microstructure in single pulses. MSP are faint objects, their single pulses normally aren't seen (the only exception is very bright nearest pulsar J0437-4715). Fortunately, the other remarkable phenomenon, "giant" pulses (GP), exceeding by hundreds or even thousands times average intensity of a pulsar, may occur in MSP: Cognard et al (1996) observed GP in J1939+2134, we suspect few other MSP. The only problem for observers is to catch sufficiently large number of pulses for representative statistic, i. e. the problem of observing time. Luckily, few radiotelescopes, which are still operating in Russia, have no full-time job at the moment.

Perhaps, MPF and GP are related phenomena. There is some indication, that they both appear at some preferable longitudes. Iikely, either are produced by the same highly excited formation in magnetosphere, aligned along field line (for instance, soliton, Mikhailovskii et al (1985)). We suspect some correlation between life-time and intensity of MPF, more intense flare lasts shorter time. 

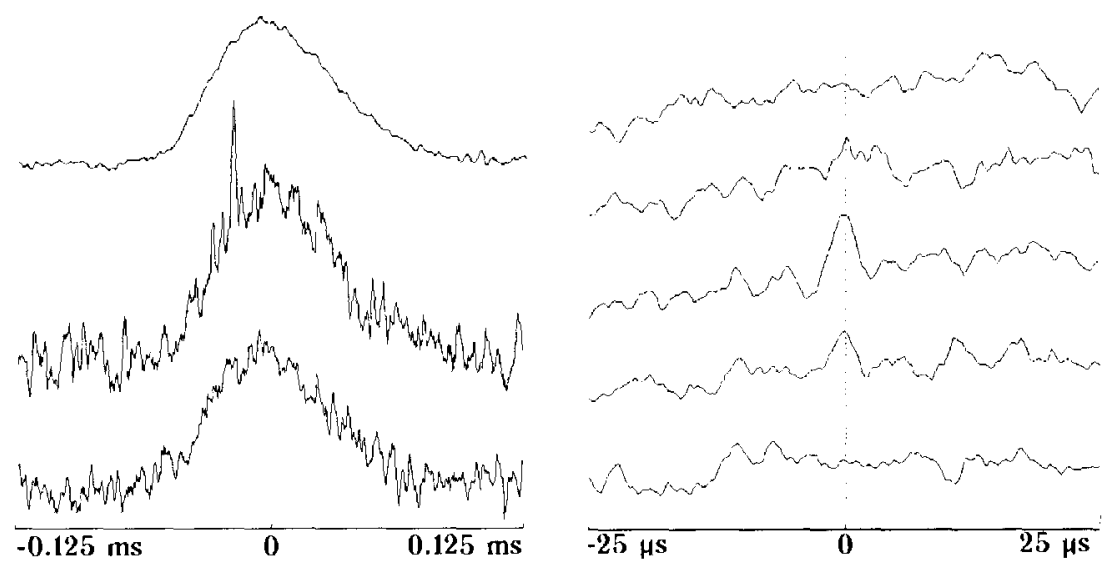

Figure 1. "Micropulse flare" in the original MSP J1939+2134, observed on 17 -th of June 1997 at $406.9 \mathrm{MHz}$. Left panel shows a mean profile of the main pulse, integrated over a large number $\left(1.8 \cdot 10^{6}\right)$ of periods (top), and short time integrations: 3 minutes during the flare (middle), the other 6 minutes, one just before and five after the flare (bottom). Right panel shows time history of the event. It displays five consequent 1 minute integrations, time runs from bottom to top. A window width is $250 \mu \mathrm{s}$ for the left panel and $50 \mu \mathrm{s}$ for the right one. On both panels resolution is $0.25 \mu \mathrm{s}$ per bin, data are smoothed by 2 us running interval.

Acknowledgments. This work is supported by RFBR, project 98-02-1697, and INTAS, grant 95-0154. The author thank the Deutsche Forschungsgemeinschaft (DFG) for the grant to support his participation in the IAU Colloquium 177.

\section{References}

Skulachev, A. D. D'Amico, N. Soglasnov, V. A. Montebulgoni, S. Cattani, A., Maccaferri, A. 1999, Astronomical Reports (submitted)

Cognard, I. Shrauner, J.A. Taylor, J. H., and Thorsett, S. E. 1996, ApJ, 457, L81

Mikhailovskii, A. B. Onishenko, O. G. and Tatarinov, E. G. 1985, Plasma Phys. and Contr. Fus., 27, 539 